\title{
Tungsten Heavy Alloy: An Alternative Plasma Facing Material in Terms of Hydrogen Isotope Retention
}

\author{
H. Maier ${ }^{a}$, R. Neu ${ }^{a, b}$, T. Schwarz-Selinger ${ }^{a}$, U. von Toussaint ${ }^{a}$, A. Manhard ${ }^{a}$, T. Dürbeck ${ }^{a}$, K. Hunger $^{a}$ \\ ${ }^{a}$ Max-Planck-Institut für Plasmaphysik, Boltzmannstraße 2, 85748 Garching, Germany \\ ${ }^{b}$ Technische Universität München, Boltzmannstraße 15, 85748 Garching, Germany \\ E-mail: Hans.Maier@ipp.mpg.de
}

Received xxxxxx

Accepted for publication $\mathrm{xxxxxx}$

Published xxxxxx

\begin{abstract}
We performed a systematic study of the deuterium retention in the tungsten heavy alloy W-2Ni-1Fe (HPM 1850 from HC Starck, Germany). Consisting of two different phases with generally two distinctly different heats of solution such alloys represent a special class of materials with respect to hydrogen isotope transport and retention. Deuterium was implanted from a plasma with an energy of $38 \mathrm{eV} / \mathrm{D}$ at sample temperatures of $100^{\circ} \mathrm{C}, 175^{\circ} \mathrm{C}$, and $250^{\circ} \mathrm{C}$ in the fluence range $10^{23} \mathrm{D} / \mathrm{m}^{2}$ to $4 \times 10^{25} \mathrm{D} / \mathrm{m}^{2}$. For each combination of implantation temperature and fluence two alloy samples were exposed together with one sample of bulk hot-rolled tungsten from Plansee SE. The amount of retained deuterium was determined by thermal desorption with heating up to $850^{\circ} \mathrm{C}$ for the alloy and $1050^{\circ} \mathrm{C}$ for bulk tungsten. The outgassed deuterium was detected by mass spectroscopy at the mass channels $3,4,19$, and 20 corresponding to $\mathrm{HD}, \mathrm{D}_{2}, \mathrm{HDO}$, and $\mathrm{D}_{2} \mathrm{O}$, respectively. The near-surface deuterium concentration was determined by ${ }^{3} \mathrm{He}$ nuclear reaction analysis. The retention in the alloy as compared to the bulk tungsten decreases systematically with increasing implantation fluence for each of the investigated implantation temperatures. For the higher fluences the ratio decreases also systematically as a function of implantation temperature.

This means that especially for high implantation fluences and temperatures the alloy HPM 1850 retains systematically less hydrogen isotopes than bulk tungsten. This property represents an important qualification criterion as a plasma-facing material for a future fusion reactor.
\end{abstract}

Keywords: Tungsten heavy alloy, plasma-facing material, hydrogen retention, nuclear reaction analysis, thermal desorption

\section{Introduction}

In 2014 the European Materials Assessment Group identified tungsten as the baseline material for state-of-the-art plasma-facing component technology for the construction of a European demonstration fusion reactor "DEMO". This choice is justified in [1] by two "key advantages", namely the high sputtering threshold, especially for hydrogen isotopes, and the low retention of tritium. At the same time the intrinsic brittleness and high ductile-to-brittle transition temperature are mentioned as high-level risks.

So-called tungsten heavy alloys would be a possible surrogate for tungsten without the problem of brittleness. For the heavy alloy $\mathrm{W}-2 \mathrm{Ni}-1 \mathrm{Fe}$ basic material property 
investigations and a first application in a present-day fusion experiment were reported in references [2-4]. In contrast to tungsten the heavy alloy is ductile and the tensile properties can even be improved with heat treatments. The thermal conductivity is fairly high. It increases from about $80 \mathrm{~W}(\mathrm{mK})^{-1}$ at $50^{\circ} \mathrm{C}$ up to $95 \mathrm{~W}(\mathrm{mK})^{-1}$ at $1200^{\circ} \mathrm{C}$. The magnetisation saturates very quickly with increasing magnetic field at very moderate levels about two orders of magnitude below that of Eurofer. The neutron activation behaviour under irradiation with a first wall neutron spectrum was computed on the basis of [5] and was found to be acceptable at least for a limited amount of material.

With respect to hydrogen isotope retention and transport tungsten heavy alloys represent a special class of materials. They consist of two phases. One phase, i.e. tungsten has a very high endothermic heat of solution for hydrogen. The other phase generally has a lower heat of solution and - due to the liquid phase sintering production - percolates throughout the whole object connecting all surfaces.

To address the issue of tritium rention in such materials, the present contribution reports on the results of a systematic study of the deuterium retention in the tungsten heavy alloy W-2Ni-1Fe (HPM 1850, HC Starck, Germany). Deuterium was implanted from a D plasma at sample temperatures between $100^{\circ} \mathrm{C}$ and $250^{\circ} \mathrm{C}$ with implantation fluences ranging from $10^{23} \mathrm{D} / \mathrm{m}^{2}$ to $4 \times 10^{25} \mathrm{D} / \mathrm{m}^{2}$. The total amount of retained deuterium was determined using thermal desorption spectroscopy. Near-surface deuterium depth profiles were determined using the $\mathrm{D}\left({ }^{3} \mathrm{He}, \mathrm{p}\right) \alpha$ nuclear reaction. Employing primary ${ }^{3} \mathrm{He}$ beam energies up to $6 \mathrm{MeV}$ yields $\mathrm{D}$ concentration profiles to a depth of about $10 \mu \mathrm{m}$.

Section 2 of this contribution presents a description of the sample material together with some thermodynamic literature data and describes the employed plasma exposure and analysis tools. In section 3 the experimental results are presented.

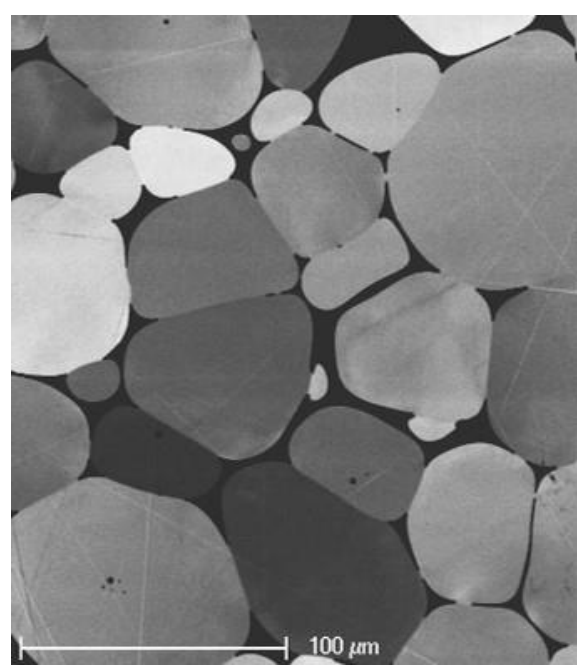

Figure 1: Backscatter electron image of a polished HPM 1850 sample surface. The scale bar at the bottom left is $100 \mu \mathrm{m}$.
Section 4 gives a discussion and interpretation of the experimental findings in view of the presented material and of accompanying numerical simulations. Finally section 5 contains a summary and conclusion.

\section{Material and experimental conditions}

\subsection{Tungsten heavy alloy}

Tungsten heavy alloys were originally proposed in 1937 for nuclear medical applications [6]. Liquid-phase sintering with copper, nickel, or mixtures thereof is proposed in the orginal publication. Technical usage as balancing weights or as heavy current circuit breaker was also already reported in [6].

\subsubsection{Microstructure}

In our work the employed material is HPM 1850 from HC Starck, Germany. This is an alloy with 2 weight $\%$ nickel and 1 weight $\%$ iron with a density of $18.5 \mathrm{~g} / \mathrm{cm}^{3}$, which is more than $95 \%$ of the density of bulk tungsten. Figure 1 shows a scanning electron microscopic image of a polished HPM 1850 sample surface in backscatter electron contrast. The image shows tungsten powder particles of strongly varying sizes with different grey levels embedded in the nickel/iron matrix shown in black. The different grey levels arise from grain orientation contrast. Although the crystal orientations are not quantifyable from the grey levels in figure 1, we can assume that each powder particle has only one crystal orientation. This is confirmed by the electron backscatter diffraction image shown in figure 2 . The image shows differently coloured tungsten particles in a $\mathrm{Ni} / \mathrm{Fe}$ matrix shown in pink. Each particle appears in only one colour, which means that it is represented by only one crystal orientation with respect to the

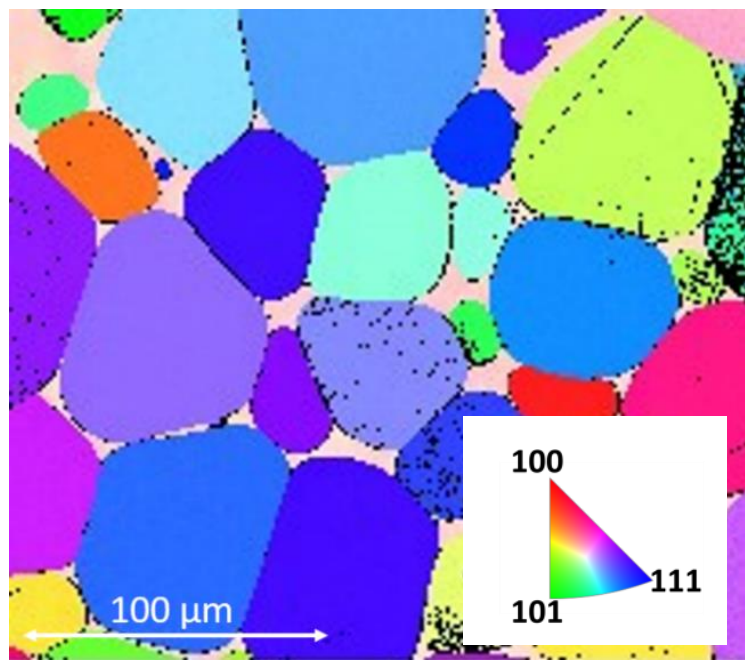

Figure 2: Electron backscatter diffraction image of a polished HPM 1850 surface. The scale bar at the bottom left is $100 \mu \mathrm{m}$. Coloured figure in online version. 
sample surface. This means that there are no crystal grain boundaries within the individual powder particles.

\subsubsection{Hydrogen thermodynamic properties}

Figures 1 and 2 show that the tungsten heavy alloy consists of two distinct phases, i.e. the particles of tungsten powder surrounded by the $\mathrm{Ni} / \mathrm{Fe}$ matrix. These represent two domains with different thermodynamic properties concerning hydrogen solution and transport.

Based on the review [7] performed by Causey in 2002, the generally accepted values for the diffusion coefficient and the solubility of hydrogen in pure tungsten are those reported by Frauenfelder in [8]. Here the diffusion coefficient

$$
D=D_{0} \times \exp \left(-\frac{E_{A}}{k T}\right)
$$

is described by a prefactor $D_{0}$ and activation energy $E_{A}$ of

$$
D_{0}=4.1 \times 10^{-7} \frac{\mathrm{m}^{2}}{\mathrm{~s}} \text { and } E_{A}=0.39 \mathrm{eV}
$$

with the Boltzmann constant $k$ and the temperature $T$, although there are indications by more recent measurements [9] and modelling [10] that the activation energy may be lower.

The second thermodynamic quantity relevant here is the heat of solution. For this reference [7] also recommends to use the value published by Frauenfelder in [8]:

$$
\Delta H_{S}^{0}=+1.04 \mathrm{eV}
$$

The other domain is the $\mathrm{Ni} / \mathrm{Fe}$ alloy phase in the ratio 2 to 1. The diffusion of hydrogen in such $\mathrm{Ni} / \mathrm{Fe}$ alloys has been studied as a function of the composition in [11]. The authors give an expression for the diffusion coefficient of hydrogen in the $\mathrm{Ni} / \mathrm{Fe}$ alloy present in HPM 1850 as:

$$
D_{0}=3.9 \times 10^{-8} \frac{\mathrm{m}^{2}}{\mathrm{~s}} \text { and } E_{A}=0.22 \mathrm{eV}
$$

There are no data available for the heat of solution in the $2: 1 \mathrm{Ni} / \mathrm{Fe}$ alloy, but it is assumed here to be in the range of those of the pure elements, which can be taken from $[12,13]$ :

$$
\Delta H_{S}^{0}(\mathrm{Fe})=+0.25 \mathrm{eV} \text { and } \Delta H_{S}^{0}(\mathrm{Ni})=+0.17 \mathrm{eV}
$$

From this comparison of literature values we can draw two conclusion:

Firstly, when we assume the heat of solution in the $\mathrm{Ni} / \mathrm{Fe}$ alloy to be similar to the values of the pure elements, we can conclude that the solution of hydrogen in the $\mathrm{Ni} / \mathrm{Fe}$ requires less energy than the solution in the tungsten powder particles.

Secondly, when we insert the values (2) and (4) into the expression (1), we can conclude that hydrogen diffusion is faster in the $\mathrm{Ni} / \mathrm{Fe}$ alloy than it is in pure tungsten for temperatures below about $600^{\circ} \mathrm{C}$.

Keep in mind that these values have been determined for hydrogen, while the experiments reported in the following have been peformed using deuterium instead and the final isotope of interest is tritium. However, in a BornOppenheimer-like adiabatic approximation the metalhydrogen interaction potential is independent of the mass of the hydrogen isotope nucleus (see for instance section 7.3 in [12] or section 5 in [14]). In reference [15] this question was investigated experimentally for hydrogen and deuterium in austenitic stainless steel. The activation energies for permeation and diffusion were found to be identical and for the diffusion coefficient an isotope effect in agreement with classical theory was found. For the diffusion of hydrogen and deuterium in tungsten identical activation energies were also found in [9].

\subsection{Experimental procedure and conditions}

After metallographic preparation of the $10 \times 10 \mathrm{~mm}^{2}$ sample surfaces [16] and outgassing in vacuum at $930^{\circ} \mathrm{C}$ for one hour the samples were exposed to a deuterium plasma. Subsequently the deuterium retention was investigated by nuclear reaction analysis and finally by thermal desorption spectroscopy. There was always a time delay of a few days between the individual steps. The setups and procedures of the individual steps are described in the following.

2.2.1 D plasma exposure. The samples were exposed to an electron-cyclotron-resonance plasma in a setup described in [17]. The ion flux to the samples consists mostly of $\mathrm{D}_{3}{ }^{+}$ions. A DC bias voltage of $-100 \mathrm{~V}$ was applied. Together with the plasma potential this corresponds to an energy of about $38 \mathrm{eV}$ per deuteron. At $38 \mathrm{eV} / \mathrm{D}$ the implantation flux is $1.25 \times 10^{20} \mathrm{D} / \mathrm{m}^{2} \mathrm{~s}$. Only $3 \%$ of the impinging ion flux are $\mathrm{D}^{+}$and $\mathrm{D}_{2}{ }^{+}$. During the implantation the samples are clamped to a temperature-controlled copper sample holder. In our experiments the implantation temperature was varied between $100^{\circ} \mathrm{C}$ and $250^{\circ} \mathrm{C}$. The implantation time varied from 20 minutes for the lowest fluence of $10^{23} \mathrm{D} / \mathrm{m}^{2}$ to four days for the highest applied fluence of $4 \times 10^{25} \mathrm{D} / \mathrm{m}^{2}$.

For each combination of implantation temperature and fluence two samples of HPM 1850 were exposed together with one tungsten reference sample to have an indication of the reproducibility. The reference material is hot-rolled polycrystalline tungsten from Plansee SE with a specified purity of $99.7 \%$ (see e.g. [18]), which was pre-treated in exactly the same way as the HPM 1850 samples. 


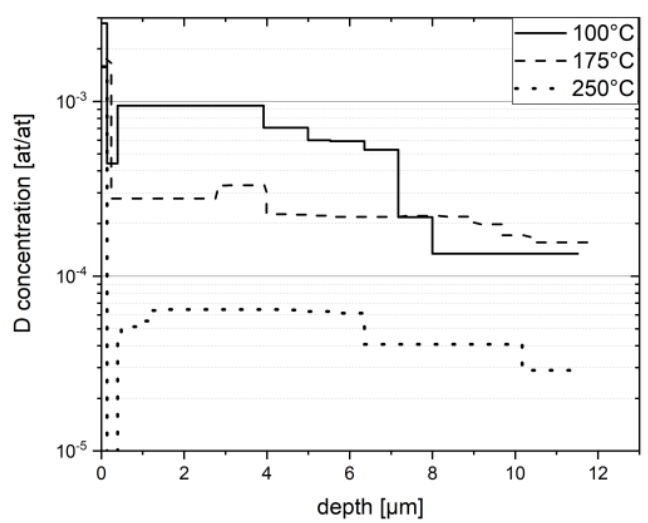

Figure 3: Deuterium concentration depth profiles in tungsten heavy alloy samples for an implantation fluence of $10^{25} \mathrm{~m}^{-2}$ at implantation temperatures of $100^{\circ} \mathrm{C}, 175^{\circ} \mathrm{C}$, and $250^{\circ} \mathrm{C}$.

2.2.2 Nuclear Reaction Analysis The near-surface deuterium content of the samples was determined by nuclear reaction analysis using the $\mathrm{D}\left({ }^{3} \mathrm{He}, \mathrm{p}\right) \alpha$ reaction and detecting the high energy protons. At the employed scattering angle of $135^{\circ}$ the cross section of this nuclear reaction has a maximum around $680 \mathrm{keV}$ in the lab system and decays to about $10 \%$ of the peak value at energies above $4 \mathrm{MeV}$ [19]. By increasing the energy of the primary ${ }^{3} \mathrm{He}$ beam this maximum can be "moved" through the sample. Using various energies up to 6 $\mathrm{MeV}$, information about the $\mathrm{D}$ content can be obtain to a depth of about $10 \mu \mathrm{m}$ in tungsten [20]. Because of the non-vanishing cross section at higher energies, i.e. lower depth, however, a deconvolution is required to construct a $\mathrm{D}$ depth profile from the data. For this the software NRADC was employed (see [21]) in combination with SIMNRA [22].

2.2.3 Thermal Desorption The thermal desorption was performed in an ultra-high vacuum setup, which basically consists of a glass tube attached to a steel vacuum chamber. The sample sits in the glass tube and is heated by a tubular furnace. Details can be found in [23]. The outgassing species are detected by a mass spectrometer. The typical base pressure is in the range of $10^{-8} \mathrm{mbar}$ in the glass tube and several $10^{-10}$ mbar in the steel chamber. During the outgassing the pressure in both chambers rose typically by a factor of 5 .

Heating is performed with a temperature ramp of the tubular furnace of $15 \mathrm{~K} / \mathrm{min}$ up to $850^{\circ} \mathrm{C}$ for the HPM samples and up to $1050^{\circ} \mathrm{C}$ for the tungsten reference samples.

In the mass spectrometer the outgassing deuterium is detected in the form of HD (mass channel 3), $\mathrm{D}_{2}$ (mass channel 4), HDO (mass channel 19), and $\mathrm{D}_{2} \mathrm{O}$ (mass channel 20). For all of these species calibration factors were determined: Calibrating $\mathrm{D}_{2}$ is straightforward using a commercially

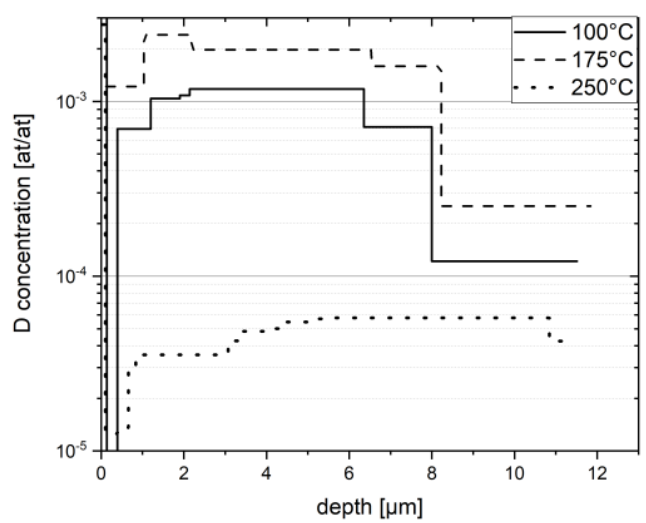

Figure 4: Deuterium concentration depth profiles in tungsten reference samples for an implantation fluence of $10^{25} \mathrm{~m}^{-2}$ at implantation temperatures of $100^{\circ} \mathrm{C}, 175^{\circ} \mathrm{C}$, and $250^{\circ} \mathrm{C}$.

available calibrated test leak. HD was calibrated using the procedure described in reference [24]. For the species HDO and $\mathrm{D}_{2} \mathrm{O}$ the measured calibration value for $\mathrm{D}_{2}$ was scaled with an overall relative sensitivity factor $\operatorname{RSF}\left(\mathrm{H}_{2} \mathrm{O}\right) / \operatorname{RSF}\left(\mathrm{D}_{2}\right)=$ $0.95 / 0.79$ according to [25] and then multiplied with the number of deuterium atoms in the corresponding molecule.

\section{Experimental Results}

The deuterium retained in the plasma-implanted samples does not represent deuterium in solid solution. After some waiting time the solute deuterium implanted during plasma exposure will have left the samples even at room temperature - what we observe is almost only deuterium retained in intrinsic trapping sites in the material [26], which can be various types of defects in the crystal lattice.

Figures 3 and 4 show examples of deuterium depth profiles for tungsten heavy alloy HPM 1850 (figure 3) as well as for

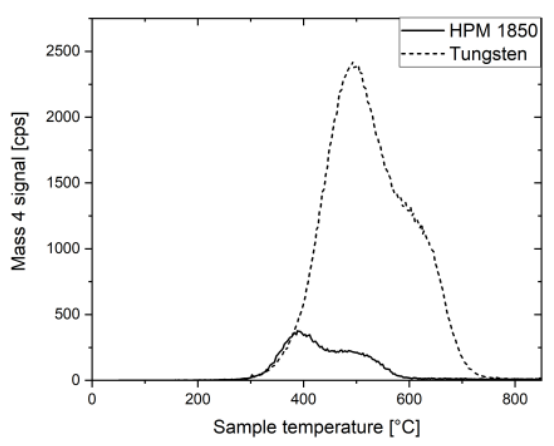

Figure 5: Thermal desorption data for tungsten heavy alloy HPM 1850 and for tungsten reference material. The plot shows the mass spectrometer signal of mass channel 4 versus the sample temperature. For both samples the implantation was done at $250^{\circ} \mathrm{C}$ to a fluence of $10^{25} \mathrm{~m}^{-2}$. 


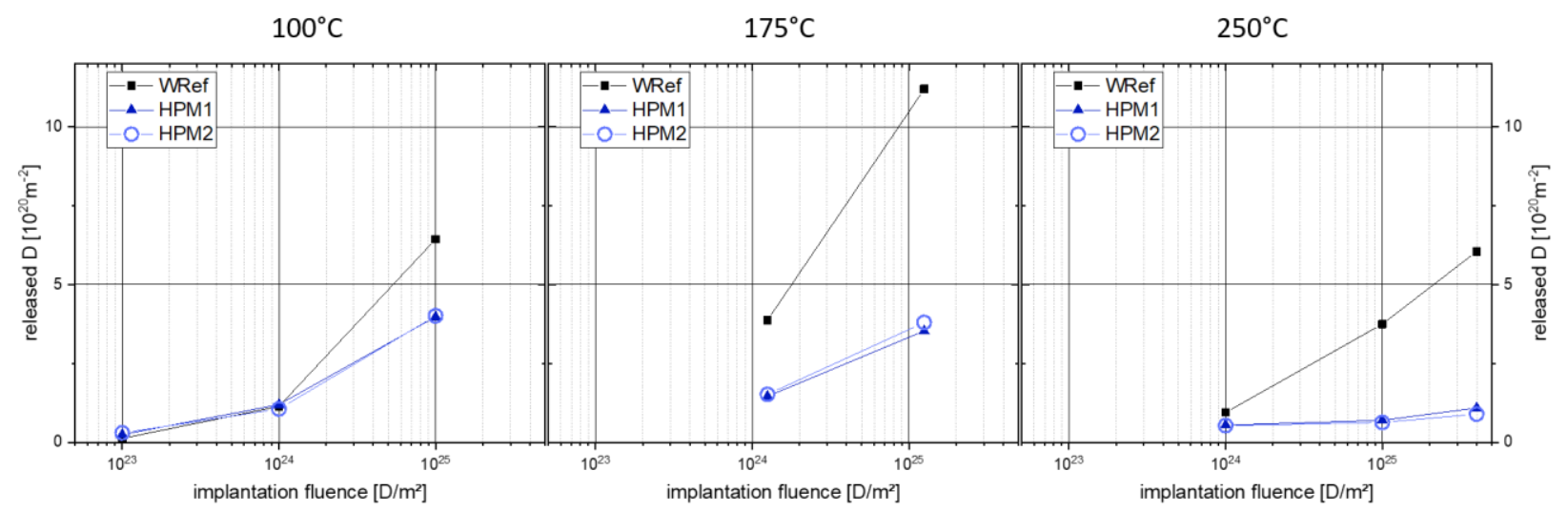

Figure 6: Total released amounts per unit area of deuterium as derived from thermal desorption as a function of the implantation fluence for implantation temperatures of $100^{\circ} \mathrm{C}$ (left), $175^{\circ} \mathrm{C}$ (centre), and $250^{\circ} \mathrm{C}$ (right). The data contain the contributions of mass channels 3, 4, 19, and 20.

the reference tungsten material (figure 4). The depth profiles are from samples with an identical implantation fluence of $10^{25} \mathrm{D} / \mathrm{m}^{2}$ and for implantation temperatures of $100^{\circ} \mathrm{C}, 175^{\circ} \mathrm{C}$, and $250^{\circ} \mathrm{C}$, respectively (see legend). Except for very nearsurface features, which may depend on the precise conditions during plasma shutdown and sample cooling, the profiles show a nearly constant deuterium concentration up to a certain depth in the material. The concentration then decays to lower values at larger depth. The maximum information depth in these experiments is about $10 \mu \mathrm{m}$.

In the tungsten heavy alloy samples the deuterium concentration in the flat part of the profiles is a monotonic function of the implantation temperature. The tungsten reference material, in contrast, shows a non-monotonic behaviour. Here the depth profile of the $175^{\circ} \mathrm{C}$ implantation shows a higher deuterium concentration at all analysed depths than the other two datasets. This is a well-known behaviour: References [27], [28] and [29] indicate that the temperature dependent deuterium retention in various tungsten materials always displays a maximum at temperatures between $100^{\circ} \mathrm{C}$ and $230^{\circ} \mathrm{C}$. At the other two temperatures we can conclude from comparing figures 3 and 4 , that the deuterium concentrations in tungsten and in the heavy alloy are very similar in the first $10 \mu \mathrm{m}$ from the surface. For the samples implanted at $250^{\circ} \mathrm{C}$ for instance, the heavy alloy as well as the tungsten reference samples show deuterium concentrations of about (4-6) $\times 10^{-5}$ in the flat parts of the depth profiles.

As explained in section 2.2 thermal desorption was performed on all samples after the nuclear reaction analysis. For the two samples displaying the lowest $\mathrm{D}$ concentrations in figures 3 and 4 , i.e. those implanted at $250^{\circ} \mathrm{C}$ to a fluence of $10^{25} \mathrm{~m}^{-2}$, figure 5 shows the mass channel 4 desorption signals, i.e. the $\mathrm{D}_{2}$ signals. The solid line represents the desorption signal from the tungsten heavy alloy HPM 1850 and the dashed line represents the desorption from the tungsten reference sample. Although the deuterium concentration depth profiles yield very similar values for the retained amount in the first $10 \mu \mathrm{m}$, the total amounts released during thermal desorption are very different. Integrating the data shown in figure 5 and adding the contributions from the other masses listed in section 2.2.3, the total deuterium amounts retained in these two samples differ by a factor of about 5 .

The total integrated deuterium amounts per unit area released in thermal desorption for all implantation temperatures and fluences are summarized in figure 6. For each implantation temperature the figure shows three data points at each implantation fluence: One data point from the tungsten reference sample (full black squares) and two data points from tungsten heavy alloy (full blue triangles and open blue circles). For all implantations these three samples were exposed together to the same plasma conditions. As the figure shows, for the tungsten heavy alloy the reproducibility of the thermal desorption measurements is excellent.

As already discussed in the frame of deuterium depth profiles, for a given fluence also the thermal desorption data show the highest deuterium retention for the tungsten reference samples implanted at a temperature of $175^{\circ} \mathrm{C}$. For a given implantation temperature the deuterium retention increases systematically with the implantation fluence. The same trend is visible for the tungsten heavy alloy. Here, however, the increase of the deuterium retention with implantation fluence becomes weaker with increasing implantation temperature. At $100^{\circ} \mathrm{C}$ the increase of deuterium retention in the tungsten heavy alloy with increasing implantation fluence is nearly identical to that in tungsten and only weakens when a fluence of $10^{25} \mathrm{~m}^{-2}$ is reached. At the highest implantation temperature investigated in this contribution, $250^{\circ} \mathrm{C}$, the deuterium retention increases only by $25 \%$ to $85 \%$ when the implantation fluence is increased by a factor of 10 to 40 . This means that the relative deuterium 
retention in tungsten heavy alloy as compared to the retention in the tungsten reference material decreases with increasing implantation fluence and temperature. At the highest values of fluence and temperature, i.e. after implantation of $4 \times 10^{25} \mathrm{D} / \mathrm{m}^{2}$ at $250^{\circ} \mathrm{C}$, the retention in the heavy alloy amounts to only $16 \%$ of that in pure tungsten.

\section{Discussion}

As shown above, the deuterium retention in tungsten heavy alloy and its relative retention as compared to pure tungsten reference material show systematic trends with increasing implantation fluence and implantation temperature. In order to explain this behaviour consistently, a common model for hydrogen in metals is considered in the following.

It is plausible to assume that the total amount of retained deuterium is controlled by two parameters: The local trap concentration and the depth into which the traps are filled. If we assume the trap concentration to be constant versus depth, then the retention is controlled solely by the diffusion depth.

When associating diffusion in a solid with a random walk process, a diffusion depth can be defined by the relation (see for instance [30]):

$$
x \sim \sqrt{D \times t}
$$

Here $x$ stands for the diffusion depth, $D$ is the diffusion coefficient, and $t$ is the time, i.e. the duration of the diffusion process. When we increase the implantation temperature, the diffusion coefficient $D$ grows exponentially, see equation 1 . This leads to an exponential growth of the diffusion depth $x$. Since the deuterium implantation is performed at a constant flux (see section 2.2.1), an increase of the implantation fluence causes an increase of the implantation duration, i.e. the time $t$ in equation 6. This also causes an increase of $x$. In this case the growth is weaker than linear.

In summary it can be concluded that a systematic dependence of the deuterium retention on implantation temperature or implantation fluence can both be traced back to a dependence on the quantity diffusion depth $x$ defined in equation 6.

Figures 3 and 4 show the near surface deuterium depth profiles for tungsten heavy alloy (3) and for tungsten (4). In both figures the data from the samples implanted at $250^{\circ} \mathrm{C}$ are the dotted lines in the lower part of the figures. The nearsurface concentration in the first ten microns is nearly the same in both cases, i.e. about a value of 4 to $6 \times 10^{-5}$. Figure 5 , however, shows the $D_{2}$ release from the two very same samples. It is quite obvious that the tungsten reference sample retained a substantially larger total amount of deuterium than the tungsten heavy alloy sample in spite of the similar local deuterium concentration in the first few microns.

Our conclusion from these data is that the excess deuterium of the tungsten sample must originate from traps in a larger depth than in the case of the tungsten heavy alloy sample. This assumption can resolve the seeming contradiction.

To fill up traps in a larger depth, the deuterium must be able to diffuse deeper into the tungsten reference material than it can into the tungsten heavy alloy. In our interpretation this is due to the combination of the microstructure of the heavy alloy - described in section 2.1.1 and shown in figures 1 and 2 - with the thermodynamic data described in section 2.1.2:

In our plasma loading deuterium is mainly implanted into the tungsten grains on the surface, since they represent the major fraction of the surface area. The deuterium atoms then perform a diffusive random walk. As the implantation depth is extremely small compared to the thickness of the sample material, most of them will leave the sample through the implantation surface and will go back into the gas phase. A small part of them can migrate deeper into the sample. At the latest when they have passed the first layer of tungsten particles, the deuterium atoms will come into contact with the second phase the material consists of, the nickel/iron matrix. In reality this contact may occur at an earlier stage, as the diffusion inside the tungsten particles is a random walk in three dimensions. If the heat of solution of hydrogen in the $\mathrm{Ni} / \mathrm{Fe}$ alloy is similar to those of the pure elements (and hence smaller than for the $\mathrm{W}$ particles, see section 2.1.2), then the deuterium atoms are energetically favoured to remain in the $\mathrm{Ni} / \mathrm{Fe}$ phase and will not go back into the tungsten phase. Due to the liquid phase sintering production process, in the $\mathrm{Ni} / \mathrm{Fe}$ phase they always have a quick diffusion path to either surface. This mechanism can limit the depth into which deuterium can diffuse in the tungsten heavy alloy and therefore can limit the deuterium retention in this material as compared to pure tungsten.

To confirm the potential effectiveness of the described mechanism we performed numerical simulations in two dimensions. The process of diffusion within the tungsten grains and the Fe/Ni alloy as well as the diffusion between the phases was modelled with a lattice Monte Carlo simulation which allows to take the different solubilities into account. We can define areas where the heat of solution and the activation energy for diffusion can be freely chosen. In our simulations we defined larger areas to which we attributed the heat of solution and diffusion activation energy of hydrogen in tungsten. We separated these areas by interconnected narrower channels to which we attributed a lower heat of solution in the range of the data from equation 5 and the diffusion activation energy of hydrogen in the $\mathrm{Ni} / \mathrm{Fe}$ alloy. Then we inserted a laterally constant hydrogen test concentration near the surface and let it diffuse through the 2D sample. What we found was indeed similar to the mechanism proposed above:

When the diffusion front leaves this first layer of tungstenlike patches and enters into the $\mathrm{Ni} / \mathrm{Fe}$ phase most of it propagates in the $\mathrm{Ni} / \mathrm{Fe}$ phase back to the surface. 
In deeper lying tungsten-like areas the concentration always remains close to zero. This numerical simulation result confirms the possible functionality of the mechanism described above.

In summary we have identified a tungsten-based material with an intrinsic limitation mechanism for hydrogen isotope retention. The mechanism is based on the presence of a second phase with a heat of solution for hydrogen isotopes, which is lower than that of the tungsten powder grains.

\section{Summary and Conclusions}

We performed a systematic study on the deuterium retention of the tungsten heavy alloy HPM 1850 in comparison to a bulk tungsten reference material. HPM 1850 is a two-phase composite material consisting of tungsten powder particles in a nickel/iron matrix. It has a density of $18.5 \mathrm{~g} / \mathrm{cm}^{3}$ and is produced by liquid-phase sintering. Therefore the $\mathrm{Ni} / \mathrm{Fe}$ phase percolates throughout the whole object.

We implanted deuterium at sample temperatures of $100^{\circ} \mathrm{C}$, $175^{\circ} \mathrm{C}$, and $250^{\circ} \mathrm{C}$ with fluences up to several $10^{25} \mathrm{~m}^{-2}$. The deuterium retention was investigated by nuclear reaction analysis depth profiling using the $\mathrm{D}\left({ }^{3} \mathrm{He}, \mathrm{p}\right) \alpha$ nuclear fusion reaction with primary ${ }^{3} \mathrm{He}$ energies up to $6 \mathrm{MeV}$. This yields an information depth of about $10 \mu \mathrm{m}$ in tungsten. Subsequently the total deuterium content of the samples was analysed by thermal desorption spectroscopy.

In tungsten we find a maximum of the deuterium retention at $175^{\circ} \mathrm{C}$. This is observed in the deuterium depth profiles as well as in the total amounts of deuterium released in thermal desorption. In comparison to tungsten the tungsten heavy alloy HPM 1850 shows a systematic decrease of the relative deuterium retention with increasing implantation fluence and also with increasing implantation temperature. At the highest implantation temperature of $250^{\circ} \mathrm{C}$ the fluence-dependent

[1] D. Stork, P. Agostini, J. L. Boutard et al. 2014 J. Nucl. Mater. 455277

[2] M. Li, D. Ruprecht, G. Kracker et al. 2018 J. Nucl. Mater. 5121

[3] R. Neu, H. Maier, M. Balden et al. 2017 Fus. Eng Des. 124 450

[4] R. Neu, H. Maier, M. Balden et al. 2018 J. Nucl. Mater. 511567

[5] R.A. Forrest, A. Tabasso, C. Danani et al. 2009 Handbook of activation data calculated using easy-2007, UKAEA FUS 552, Culham Science Centre, EURATOM/UKAEA Fusion Association, United Kingdom

[6] C. J. Smithells 1937 Nature 139490

[7] R. A. Causey 2002 J. Nucl. Mater. 30091

[8] R. Frauenfelder 1968 J. Chem. Phys. 483955

[9] G Holzner et al 2020 Phys. Scr. T171 014034

[10] Heinola K and Ahlgren T 2010 J. Appl. Phys. 107113531 increase of the deuterium retention in the heavy alloy is very weak and increases only by $25 \%$ to $85 \%$ when the implantation fluence is increased by a factor of 10 to 40 . After implantation of $4 \times 10^{25} \mathrm{D} / \mathrm{m}^{2}$ at $250^{\circ} \mathrm{C}$, the retention in the heavy alloy amounts to only $16 \%$ of that in pure tungsten.

We attribute this to the presence of the $\mathrm{Ni} / \mathrm{Fe}$ phase in the material. It has a lower heat of solution than pure tungsten and provides diffusion paths to the surface.

In conclusion we have identified a class of materials which provide an intrinsic limitation mechanism for the retention of hydrogen isotopes. Due to its higher heat of solution tungsten in the bulk of the materials is not accessible for hydrogen isotopes. Therefore trapping of tritium in deeper layers of the sample cannot occur - irrespective of the number of traps existing in the tungsten phase.

Together with the good mechanical properties of tungsten heavy alloys this specific property could be extremely beneficial for constructing plasma-facing components in fusion reactors with a low retention of tritium. It remains to be seen how this property develops under neutron irradiation.

\section{Acknowledgements}

We would like to thank J. Dorner and M. Fußeder at MaxPlanck-Institut für Plasmaphysik for their support in accelerator operation and K. Schmid for support with the use of NRADC.

This work has been carried out within the framework of the EUROfusion Consortium and has received funding from the Euratom research and training programme 2014-2018 and 2019-2020 under grant agreement No 633053. The views and opinions expressed herein do not necessarily reflect those of the European Commission.

\section{References}

[11] R. Dus and M. Smialowski 1967 Acta Metallurgica 15 1611

[12] Y. Fukai 2005 The Metal-Hydrogen System, Springer, $2^{\text {nd }}$ edition

[13] E. Fromm and G. Hörz 1980 Int. Metals Rev. 25269

[14] S. M. Myers et al. 1992 Rev. Mod. Phys. 64559

[15] N. R. Quick and H. H. Johnson 1979 Metallurgical Transactions A 10A 67

[16] K. Hunger et al. 2020 54. Metallographie-Tagung Saarbrücken, Germany

[17] A. Manhard et al. 2011 Plasma Sources Sci. Technol. 20 015010

[18] A. Manhard et al. 2015 Practical Metallography 52437

[19] B. Wielunska et al. 2016 Nucl. Inst. Meth. B 37141

[20] M. Mayer et al. 2009 Nucl. Inst. Meth. B 267506

[21] K. Schmid, U. von Toussaint 2012 Nucl. Inst. Meth. B

28164 
[22] M. Mayer 1997 SIMNRA User's Guide, Report IPP 9/113, Max-Planck-Institut für Plasmaphysik, Garching, Germany

[23] E. Saloncon et al. 2008 J. Nucl. Mater. 376160

[24] P. Wang et al. 2013 Nucl. Inst. Meth. B 30054

[25] Hiden Analytical Application Note 282, www.hiden.de
[26] J. Roth and K. Schmid 2011 Phys. Scr. T145 014031 [27] V. Kh. Alimov et al. 2009 Phys. Scr. T138 014048 [28] V. Kh Alimov et al. 2012 J. Nucl. Mater. 420519 [29] A. Manhard, Dissertation Universität Augsburg 2011 [30] H. Mehrer 2007 Diffusion in Solids (Springer Series in Solid State Sciences, Springer, Berlin) p 155 